\title{
Fuzzy Logic based Adaptive Noise Filter for Real Time Image Processing Applications
}

\author{
Jasdeep Kaur \\ Student of M,tech,Bhai Maha Singh College of \\ Engineering,Shri Muktsar sahib
}

\author{
Preetinder Kaur \\ A.P,Bhai Maha Singh \\ College of Engineering,Shri Muktsar sahib
}

\begin{abstract}
In this paper we implement a new technique for detection and removal of impulse noise from the grayscale digital images. Proposed method consist of the three steps, in the first step, center pixel of the window is tested whether impulse noise is present or not, detected pixel has impulse noise when it lies outside from the trimming range by using fuzzy reasoning. In the second step, we replace the noisy pixels by using median filters. In third stage we create a histogram if the image and again remove the noise by using soft thresholding. The results of the proposed technique for the removal and detection of impulse noise from the gray scale images is very good rather than exiting technique in terms of PSNR values.
\end{abstract}

\section{Keywords}

Impulse noise, Median filter, Soft thresholding, Fuzzy logic, Histogram

\section{INTRODUCTION}

Whenever an image is converted from one form to another such as, digitizing, scanning, transmitting, storing, etc., some of the degradation occurs at the output.[1] Hence, the output image suffer from a special kind of noise "salt \& paper". Salt \& paper significantly degrades the image quality [2]. An image denoising is used to remove the additive noise. The goal of image denoising is to estimate the original image from the noisy image.Denoising is one of the important task and pre-processing step in digital image processing. There are many median filters are available for impulse noise reduction although these methods have been improved, but the quality of denoising image is still not satisfactory [6]. But this method is too time-consuming. Most of these algorithms provide suitable and good results at smaller percent of noise levels and find difficulty with higher level noises, also this method is too time-consuming and isn't suitable for real applications[6].we implement the new technique is better than the exiting technique. This approach has been adopted to remove noise from 'TV signal and to reduce interlaced scan related artifacts at the receiver side. Moreover, an algorithm for image quality enhancement has been developed which tunes locally the contrast and the brightness of the picture with respect to the global image characteristics[3]. The proposed technique can work in low time and have better results in PSNR metric. It introduces the artifacts and blurring of the images. So it is a challenge for the researchers to remove the impulsive noise from the gray scale images while retaining the important signal features and explore the possibilities of various denoising technique for gray scale images.

\section{Impulse noise:}

A grayscale image represented by a two-dimensional array where a location $(i, j)$ is a position in image and called pixel. Often the grayscale image is stored as an 8-bit integer that giving 256 possible different shades of gray going from black to white, pixels can have value in [0-255] integer interval, but some pixels in an image have not correct value and they are noise that their value's is 0 or 255 , thus we can present below model for a grayscale image that have impulse noisy pixel in relation (1).[1]

$$
\operatorname{img}(i, j)=\left\{\begin{array}{cl}
O R G(i, j) & \text { with probability } 1-p r \\
0 & \text { with probability } p r_{1} \\
255 & \text { with probability } p r_{2}
\end{array}\right.
$$

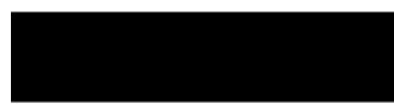

0

$[0,255]$

Figure 2.1 Representation of impulse noise (Salt \& Pepper Noise )[4].

\section{Median Filters:}

Median filter is used to remove the disadvantage of the mean filter. This filter is mainly used to reduce impulse noise from the gray scale images. The nature of this type of filter is nonlinear. In image processing, several filtering algorithms belong to a category called windowing operators. Windowing operators use a window, or neighborhood of pixels, to calculate their output. For example, windowing operator may perform an operation like finding the average of all pixels in the neighborhood of a pixel. The pixel around which the window is found is called the origin.

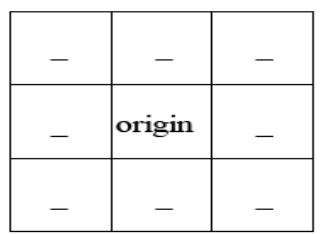

Figure 3.1 pixel window and origin [5]. 


\section{Fuzzy Logic Based Algorithms for Image Processing:}

As far as noise is concerned the proposed filters have been designed to reduce respectively impulsive and Gaussian noise. The first one uses three cascaded fuzzy processes, which analyze the four directions into a $(3 \times 3)$ or $(3 \times 2)$ or $(4 \times 4)$ window centered on the pixel to be processed. The complete fuzzy system is tuned in order to preserve the motion information and one pixel wide edges. The output of the last fuzzy stage provides an evaluation of the pixel noisiness. on the ground of which a median filter is weighted. Conceding the Gaussian noise, the classic temporal recursive average filter has been optimized with a fuzzy motion detector [3]. This one uses fuzzy rules operating on a measure of the spatial and temporal pixel correlation in the vertical and diagonal directions of the $(3 \times 3)$ or $(3 \times 2)$ or $(4 \times 4)$ window. The motion detector selects the direction where the pixels are more correlated temporally and spatially: this information is used to compute the new pixel value resulting from the weighted average of the original pixel and the other window points in the selected direction. Here again motion information is unaffected by filtering. The same fuzzy motion detector has been used for the scanning rate up conversion. In this case if the pixel is still no interpolation is performed. Otherwise a weighted average between a three point vertical median filter output and the selected direction pixels is used. A better image quality can be obtained by adding a fuzzy filter to adjust locally the luminance dynamic range and enhance picture details (Fuzzy- Contrast Luminance Controller or FCLC). This filter allows recovering details that are attenuated by dynamic range mismatches between the original and the displayed image. The filter is composed by two fuzzy processes respectively modifying the luminance range and enhancing the high frequency [3].

\section{Implementation:}

The implementation of the improved median filtering algorithm is used to reduce noise in the image. The simple idea is to examine the pixel values in the selected window of the input signal and replace the noisy and distorted or blurred pixels with the median/effective median[5].The program works by using a moving fixed $3 \times 3$ window of pixel neighborhoods. Then use the $3 \times 2$ and $4 \times 4$ window to remove the noise from the edges of the image also create the histogram of image. The proposed algorithm achieves very good results then the exiting algorithm.

The steps of the proposed algorithm are described as below:

Step1. Read the gray scale image: First of all read the gray scale image in the MATLAB software. The proposed technique is Used the Lena gray scale image to compare the results of proposed technique with the existing technique. 1 will show in the fig 4.1 .

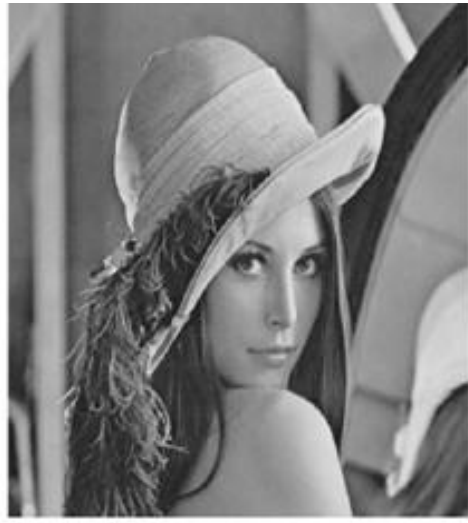

Figure 5.1 Original gray scale Lena image.

Step 2: Add the "salt pepper" noise to the image. In order to test the performance rate of this proposed algorithm experiments are Performed at different noise levels ranging from 5\% to $40 \%$ on two types of images. The two images Lena and cameraman are two dimensional 8-bit grayscale images. Impulse noise of different percentages ranging from $5 \%$ to $40 \%$ is added to the two types of images. Intensive simulations were carried out on the two polluted images with different percentages of impulse noise (salt and pepper).it show fig 3(b).

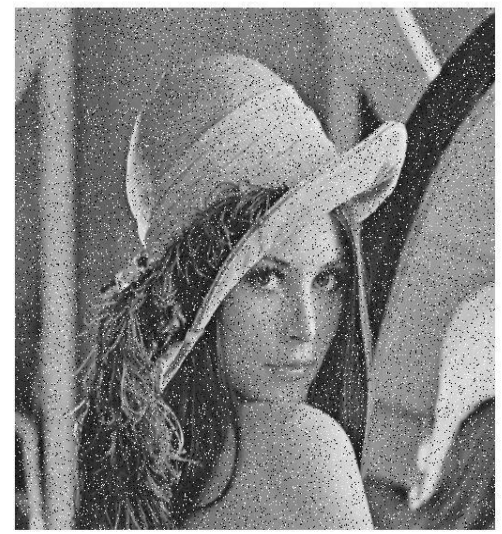

Figure 5.2. Lena image corrupted by $40 \%$ impulse noise.

\section{Step 3. 3x3 median filters are used to remove the noise from the gray scale noisy image.}

In the third step $3 \times 3$ matrix scanning fuzzy logic based median filtering. The neighboring pixels are ranked according to brightness (intensity) and the median value becomes the new value for the central pixel. Median filters can do an excellent job of rejecting certain types of noise, in particular, "shot" or impulse noise in which some individual pixels have extreme values. In this filtering operation, the pixel values in the neighborhood window are ranked according to intensity and the middle value (the median) become the output value for the pixel under evaluation. so the process is apply to the $3 \times 3$ matrix scanning fuzzy logic based median filter to the noisy gray scale image. It remove the noise from the previously generated noisy image as figure 5.2.After $3 \times 3$ matrix Scanning it show the result as in the figure 5.3.The scanning of the $3 \times 3$ median filters on the gray scale Lena 
image. It will scan the image as to reduce the impulse noise from the image. Process is explained as below:

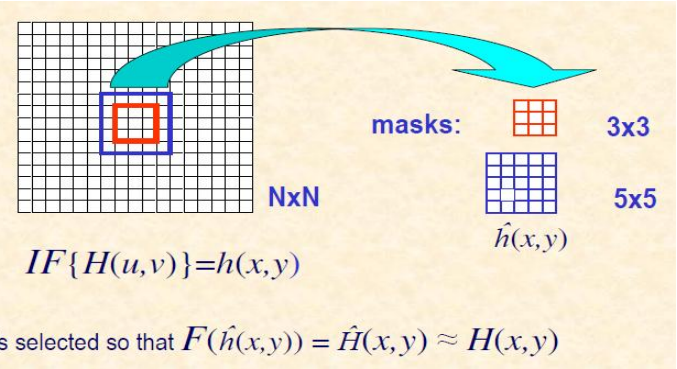

\section{Figure 5(a) represents the Filter definition in spatial domain.}

The figure5(a) represents the filtering process of the $3 \times 3$ median windows which is the sub window of the $5 \times 5$ window.Centre pixel of the $3 \times 3$ window is selected to identifying wither it is noisy or not.

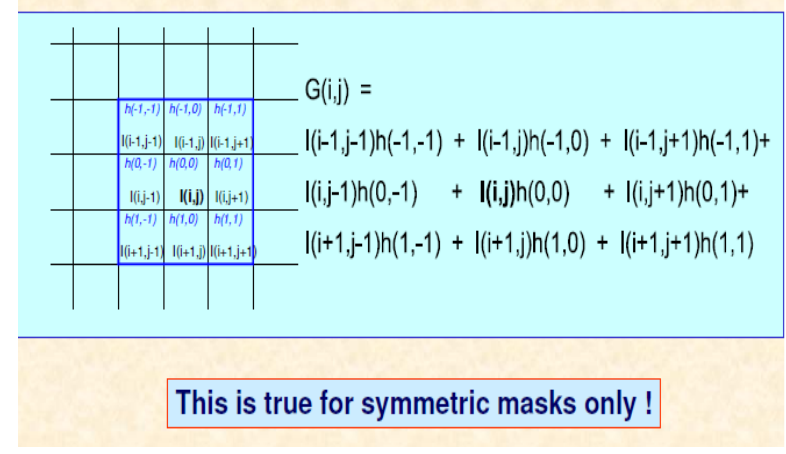

Figure 5(b) image and filter convolution

The figure5(b) represents the scanning process by taking the center pixel of the $3 \times 3$ matrix window for the removal of the noise from the gray scale images. This figure represents the scanning process of rows and columns of the image. It means that it scan the centre pixel of the window by considering the neighborhood value. Basically this process is used in the median filters to decide the pixel is noisy or not if it has noise than discard the noisy pixel and replace it by calculating the average value of the neighboring pixel.

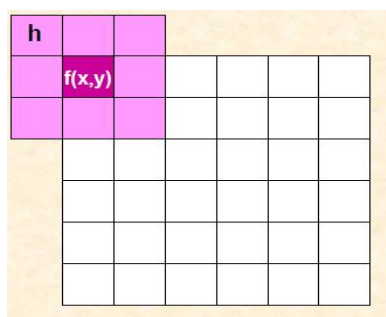

source image $f$

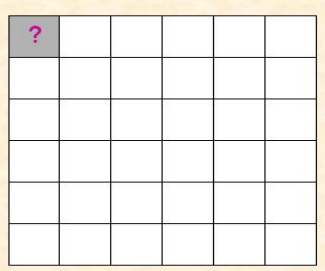

output image $g$
Figure 5(c) represents the boundary effects.

The figure 5(c) represents the what kind of boundary effects can be on the gray scale image by using $3 \times 3$ median filters, I can also say that wither it remove the noise on the boundaries or not. It represents the what is the output of the $1^{\text {st }}$ pixel by taking the $3 \times 3$ matrix window.
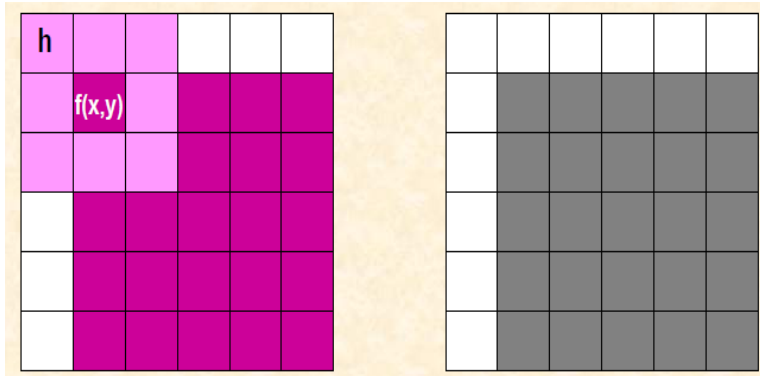

Boundary columns and rows of $(\mathrm{NxN})$ image are neglected and the filtered image is of size $(\mathrm{N}-2) \times(\mathrm{N}-2)$

\section{Figure 5(d) represents the boundary effects by using $3 \times 3$} median filter.

The $3 \times 3$ median filter windows put the effects on the boundary of the gray scale image. This is the disadvantage of the $3 \times 3$ median filters because it can scan the image up to the columns-2 and row-2, therefore boundary columns and rows are neglected.

Step 4: In the step $43 \times 2$ matrix scanning fuzzy logic based median filter to the noisy gray scale image. Process is same as in the previous step but in the previous $3 \times 3$ scan matrix has some disadvantage that it remove the noise from the centre of the image but some kind of noise is remain on the edges of the image. So to remove the noise from the edges of image $3 \times 2$ matrix scanning is used. It apply this procedure in fig 5.2.it produce the better quality image and resultant image is fig 5.3.

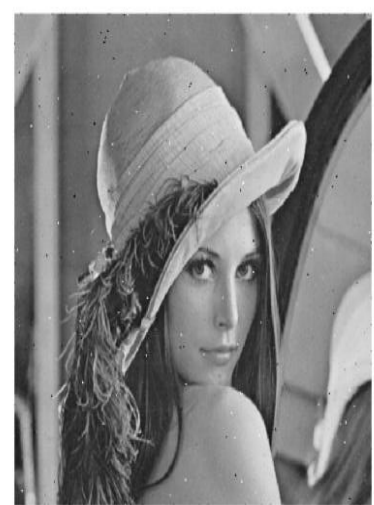

Figure 5.3 this image represent the filter outputs of the $3 \times 3$ matrix scanning fuzzy logic based.

Step 5: Again to improve the image quality $4 \times 4$ matrix scanning fuzzy logic based median filter is used and create the histogram also. Histogram is creating to show the remaining noise in the image. It show in the fig 5.4. Same process is repeated again for the $4 \times 4$ matrix scanning for the removal of noise by using fuzzy based median filter. As like in the $3 \times 3$ median filter it scan the matrix upto row-2,and col- 2 only.and in the process of $3 \times 2$ medain filter which is fuzzy based is used only for the boundary scanning matrix. So now at last $\mathrm{i}$ have applied the $4 \times 4$ to improve the image quality more and it will scan the matrix upto row-3,col-3 to remove the noise from the gray scale corrupted Lena image. 

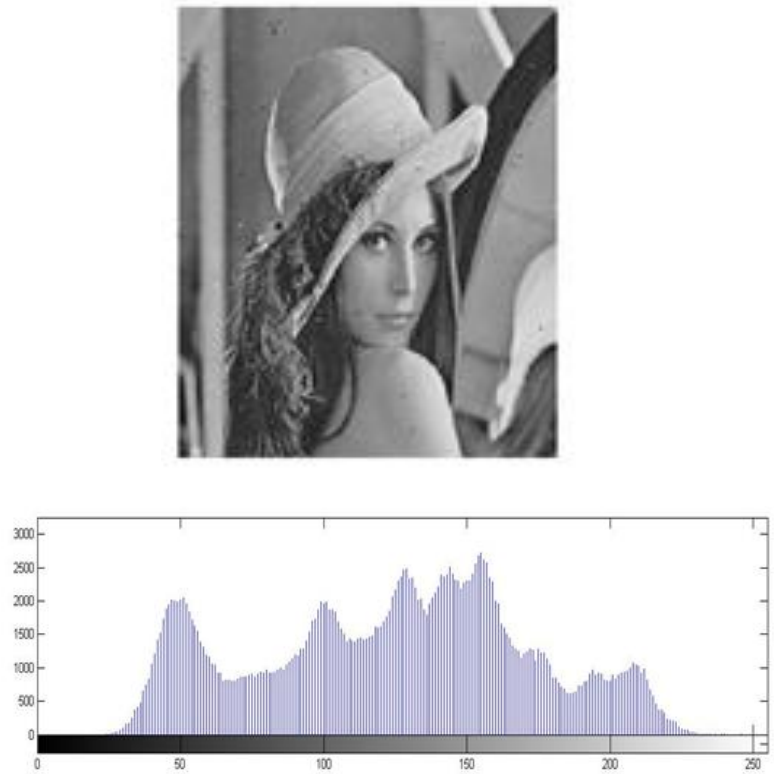

Figure 5.4 after apply the $3 \times 2$ and $4 \times 4$ matrix scanning fuzzy logic based and create histogram of the image.

Step 6: Remaining noise is removed from the image by creating histogram of the image.
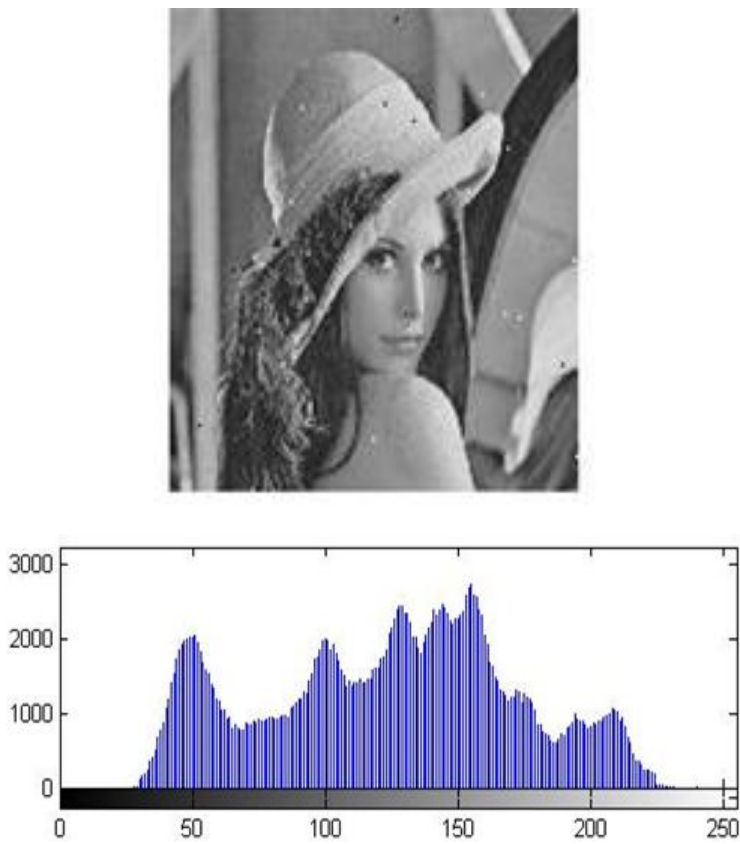

Figure 5.5 represent the better quality image and remaining noise is filtered by using the soft thresholding concept.

\section{RESULTS:}

In order to test the performance rate of this proposed algorithm experiments are performed at different noise levels ranging from $5 \%$ to $40 \%$ on Lena image. The lena image is 8 bit grayscale image. Impulse noise of different percentages ranging from 5\% to $40 \%$ is added to lena image. the adaptive median filter which is based on the fuzzy logic is applied to the corrupted images by impulse noise. The performance of the proposed algorithms was evaluated in terms of the visual quality, the peak-signal-to-noise-ratio (PSNR) and the stability of the performance of filters on different types of images. The experiment was carried out to study the performance of the detection schemes in identifying the noisy pixels in the Lena image at different impulse noise ratios[5].

PSNR $=10 \log 10\left(R^{2} / \mathrm{MSE}\right)[5]$

$$
\mathrm{MSE}=\frac{1}{M N} \sum_{i=1}^{M} \sum_{j=1}^{N}[Y(i, j)-\hat{Y}(i, j)]^{2}
$$

We analysis this method with PSNR (Pick Signal Noise Ratio) metric and visual comparison, the results show this method is very good for noise reduction and image restoration in high level noisy images.

Detection performance on the lena image for random variable impulse noise as follow:

Table 1: comparison of the results of Existing technique with the Proposed method technique filter for the Lena image.

\begin{tabular}{|l|l|l|l|l|l|l|l|l|}
\hline percentage & 5 & 10 & 15 & 20 & 25 & 30 & 35 & 40 \\
\hline $\begin{array}{l}\text { Existing } \\
\text { method(psnr) }\end{array}$ & $\begin{array}{l}35 . \\
77\end{array}$ & $\begin{array}{l}34.6 \\
1\end{array}$ & $\begin{array}{l}33.4 \\
6\end{array}$ & $\begin{array}{l}32.5 \\
7\end{array}$ & $\begin{array}{l}31.7 \\
4\end{array}$ & 30 & 29 & 28 \\
\hline $\begin{array}{l}\text { Proposed } \\
\text { method(psnr) }\end{array}$ & $\begin{array}{l}42 . \\
60\end{array}$ & $\begin{array}{l}41.2 \\
5\end{array}$ & $\begin{array}{l}39.9 \\
8\end{array}$ & $\begin{array}{l}38.5 \\
7\end{array}$ & $\begin{array}{l}36.4 \\
9\end{array}$ & $\begin{array}{l}.35 .5 \\
9\end{array}$ & $\begin{array}{l}33.1 \\
1\end{array}$ & $\begin{array}{l}32.0 \\
9\end{array}$ \\
\hline $\begin{array}{l}\text { Proposed } \\
\text { method(mse) }\end{array}$ & 3.5 & 4.90 & 6.98 & 9.08 & $\begin{array}{l}14.6 \\
8\end{array}$ & $\begin{array}{l}18.0 \\
8\end{array}$ & $\begin{array}{l}31.9 \\
7\end{array}$ & $\begin{array}{l}40.4 \\
7\end{array}$ \\
\hline
\end{tabular}

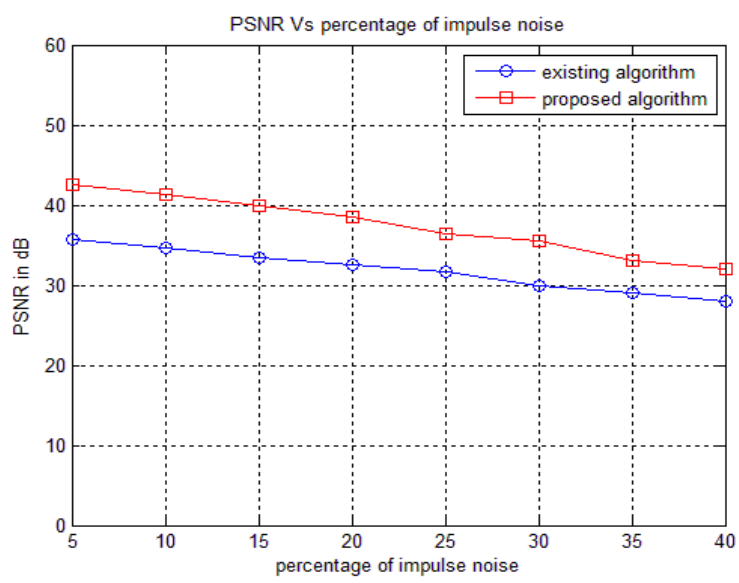

Figure 4.1: Variation of PSNR with respect to the impulse-noise percentage for different values on the Lena image for random-valued impulse noise.

The graph shows the variation of PSNR with respect to impulse noise for the different values on the Lena image for the random valued impulse noise. PSNR is varying with the percentage of the impulse noise. The above graph shows the comparison of the exiting algorithm and the proposed algorithms. In order to test the performance rate of this proposed algorithm experiments are performed at different noise levels ranging from $5 \%$ to $40 \%$ on Lena image. The Lena image is 8-bit grayscale image. Impulse noise of different percentages ranging from $5 \%$ to $40 \%$ is added to 
Lena image. The adaptive median filter which is based on the fuzzy logic is applied to the corrupted images by impulse noise.

\section{CONCLUSION AND FUTURE SCOPE:}

It have been concludes that fuzzy logic based on adaptive noise filtering technique gives the better results as compared to the exiting technique. It detects and removes impulse noise in gray scale digital images. We detect noisy pixels by using fuzzy reasoning with lowest uncertainty and we replace noisy pixels with a median filter. After then remaining noise is removed by creating the histogram of that image by using soft thresholding method. We analysis this method with PSNR (Pick Signal Noise Ratio) metric and MSE (mean square error) and visual comparison, the results show this method is very good for noise reduction as compared with the exiting technique.

FUTURE SCOPE: In this thesis, noise detection is mostly covered and for noise filtration median filter is used. Research may be undertaken to devise better filtration techniques. This technique together with a best detection technique can result in optimal restoration of degraded image. As it has been stated that the existing as well as proposed techniques are computationally expensive, investigation may be carried out in this direction. The future plan of the proposed method is to extend it further for removing impulse noise from color images.

\section{REFERENCES}

[1] Mahdi Jampour and Mehdi Ziari, 2010 Impulse noise Detection and Reduction using Fuzzy logic and Median Heuristic Filter, International Conference on Networking and Information Technology.
[2] Tonghan Wang, Xingyi Li, 2011. An Efficient Impulse Noise Reduction Algorithm.

[3] M. Mancuso, R. Poluzzi, and GG. Rizzotto 1992, Filter Architecture Particularly for Video Applications".

[4] Pankaj Kumar Sa,2006.On the Development of Impulsive Noise Removal Schemes,Department of Computer Science and Engineering National Institute of Technology,Rourkela-769 008, Orissa, India.

[5] Weyori, Benjamin Asubam,2011,improved median filtering algorithm for the reduction of impulse noise in corrupted $2 \mathrm{~d}$ greyscale images, a thesis submitted to the department of computer engineering Kwame Nkrumah university of science and technology in master of philosophy.

[6] Jasdeep kaur, Pawandeep kaur and Preetinder kaur mann (july 2012) "Review of impulse noise reduction technique using fuzzy logic for the image processing", International journal of engineering and technology ,vol.1,issue 5 .

[7] ZHANG Hong-qiao, MA Xin-jun, and WU-Ning, “A New Filter Algorithm of Image Based on Fuzzy Logical",IEEE, pp. 315-318, 2011

[8] Aborisade, D.O “ A Novel Fuzzy logic Based Impulse Noise Filtering Technique", International Journal of Advanced Science and Technology Vol. 32, July, 2011. 